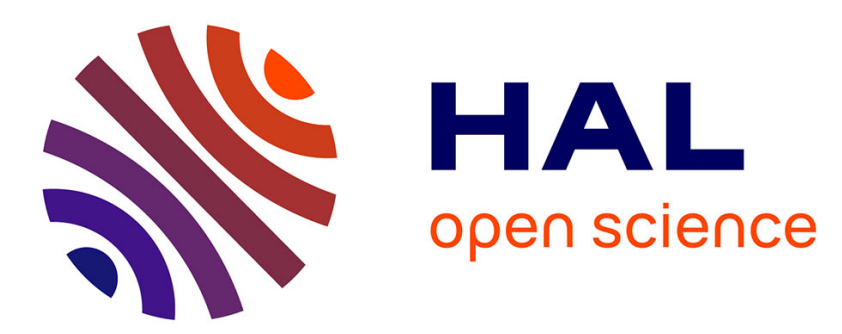

\title{
Mechanism of Enhanced Triplet Decay of Thionucleobase by Glycosylation and Rate-Modulating Strategies
}

\author{
Shuming Bai, Mario Barbatti
}

\section{To cite this version:}

Shuming Bai, Mario Barbatti. Mechanism of Enhanced Triplet Decay of Thionucleobase by Glycosylation and Rate-Modulating Strategies. Physical Chemistry Chemical Physics, 2018, 20 (24), pp.16428-16436. hal-01965457

\section{HAL Id: hal-01965457 \\ https://hal-amu.archives-ouvertes.fr/hal-01965457}

Submitted on 26 Dec 2018

HAL is a multi-disciplinary open access archive for the deposit and dissemination of scientific research documents, whether they are published or not. The documents may come from teaching and research institutions in France or abroad, or from public or private research centers.
L'archive ouverte pluridisciplinaire HAL, est destinée au dépôt et à la diffusion de documents scientifiques de niveau recherche, publiés ou non, émanant des établissements d'enseignement et de recherche français ou étrangers, des laboratoires publics ou privés.

\section{(c)(1)}

Distributed under a Creative Commons Attribution| 4.0 International License 


\title{
Mechanism of Enhanced Triplet Decay of Thionucleobase by Glycosylation and Rate-Modulating Strategies
}

\author{
Shuming Bai* and Mario Barbatti* \\ Aix Marseille Univ, CNRS, ICR, Marseille, France
}

\section{Abstract}

The decay of the triplet state of photosensitizers is essential to their performance in singlet-oxygen generation. Experiments have shown that in thionucleosides this decay is enhanced compared to that in the corresponding thionucleobases. In this work, we applied quantum-chemical methods and chemical-kinetic modeling to investigate the effects of sugar substituent on the triplet decay of thionucleosides. The computed rates for the energetically favored conformers of thiothymidine, thiouridine, and thioguanosine (and the respective thionucleobases) show a remarkable quantitative agreement with the experimental results. We additionally show that the triplet decay enhancement is caused by the repulsion interaction between the sugar group and the sulfur atom, which reduces the activation energy for intersystem crossing, by destabilizing the $T_{1}$ minimum. In some instances, an intramolecular hydrogen bond stabilizes the energy of the $\mathrm{T}_{1} / \mathrm{S}_{0}$ crossing point, also reducing the activation energy. This molecular understanding of the mechanism of enhanced triplet decay provides a guideline to control the triplet decay rate, which was tested in new thiothymidine derivatives. 


\section{Introduction}

Photoinduced processes in thio-modified nucleobase and nucleoside derivatives have been investigated in the past few decades aiming at various biotechnological and medicinal applications, including phototherapeutic agents, ${ }^{1,2}$ immunosuppressants, ${ }^{3,4}$ and initiators of photochemical transformations. ${ }^{5}$ Unlike the canonical nucleobases, where the quantum yield of triplet state after photoexcitation is always near zero, ${ }^{6,7}$ these thio-derivatives usually have triplet quantum yields near the unity, with ultrafast intersystem crossing (ISC) taking place even in the sub-picosecond scale. ${ }^{8-11}$ Moreover, the experimental results show a sharp difference between thionucleobases and their thionucleoside counterparts regarding absorption strengths, ${ }^{9}$ internal conversion lifetimes, ${ }^{12}$ and triplet decay rate. ${ }^{13}$

Concerning the triplet decay rate, this quantity plays a crucial role in the field because it ultimately controls the singlet-oxygen yield, which may, in turn, determine from the efficacy of a phototherapeutic drug to the carcinogenic side-effects of an immunosuppressant. Taras-Goslinska et al. found out that the nonradiative decay rate of the $T_{1}$ state of 2-thiothymindine (2tThd) in acetonitrile solution is about 14 times larger than the one of 2-thiothymine (2tThy); and that the rate of 5-methyl-2-thiouridine (2tThr, with ribose added to 2tThy) is 23 times larger. ${ }^{13}$ In contrast, Crespo-Hernandez' group has recently reported that the triplet decay rate ratio of 6-thioguanosine (6tGuo) is less than twice as larger than that of 6-thioguanine (6tGua). ${ }^{14}, 15$ These enhanced triplet decay rates but with significanlty different ratios raise one fundamental question: how does the sugar substituent affect the triplet decay?

Experimental research alone has not been enough to answer this question. In the mentioned research by Taras-Goslinska et al., ${ }^{13}$ different experimental techniques - 
including deuterated derivative and intermolecular quenching — were utilized, but none of them would definitely explain the enhanced triplet decay rate.

With contributions from different theoretical groups, the generation of triplet state in thionucleobases has been intensively investigated based on the excited-state topography and nonadiabatic dynamics simulations. ${ }^{16-22}$ Nevertheless, the subsequent (and more related to the clinical applications) steps, including the intrinsic decay dynamics of the $T_{1}$ triplet state and chemical reactions with other species, such as molecular oxygen, have received much less attention from theoretical studies. ${ }^{23,24}$ In a series of recent works, we have applied high-level quantum chemistry and developed chemical-kinetical models to explore both the fate of the triplet states and the singletoxygen photosensitization reaction of thionucleobases. ${ }^{25-27}$ For the intrinsic decay of their triplet state, we proposed a two-step mechanistic model based on the two $T_{1}$ minima characterizing the topography of this state. The energetic balance between these minima determines the decay rate of triplet state, with ISC happening due to coupling to the $\mathrm{S}_{0}$ state near the sulfur out-of-plane (op_S) minimum. ${ }^{25}$
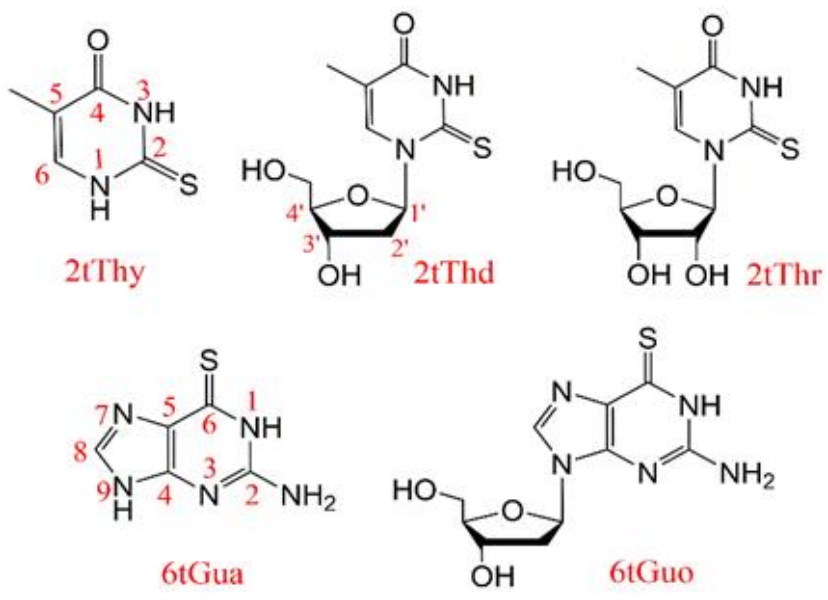

Figure 1. Structure of the investigated molecules: 2-thiothymine (2tThy), 2-thiothymindine (2tThd), 5-methyl-2-thiouridine (2tThr), 6-thioguanine (6tGua), and 6-thioguanosine (6tGuo). 
The theoretical research so far has been mostly restricted to thionucleobases, without considering either ribose or deoxyribose sugar groups. As a result, little is known about the molecular mechanisms underlying the photophysics of thionucleosides. In this work, we close this knowledge gap. Our goal is to answer why the sugar substitution enhances the triplet decay rate in thionucleosides, and why the ratio of this enhancement is much more significant on thiopyrimidines than on thiopurines. We accomplished that based on quantum chemical analysis of thionucleobases and their corresponding thionucleosides, including the pyrimidines 2tThy, 2tThd, and 2tThr, and the purines 6tGua and 6tGuo. (Their structures are shown in Figure 1.) Then, a general mechanism to control the triplet decay rate is proposed and tested for specially designed new thioderivatives.

\section{Computational details}

\subsection{Electronic structure calculations}

During our previous research on thionucleobases, we found out that, for describing the $\mathrm{T}_{1} / \mathrm{S}_{0}$ ISC of $2 \mathrm{t}$ Thy from the op_S minimum, the quality of the density functional theory (DFT) based on a hybrid functional was nearly the same as the much more expensive multistate multireference perturbation theory to second $\operatorname{order}^{28}$ (MS-CASPT2). Direct DFT calculation of $T_{1}$ open-shell ground-state was also found to perform better than the computation of $\mathrm{T}_{1}$ with the linear-response time-dependent theory (TDDFT) ${ }^{25}$ Taking this previous experience into account, here we follow an economical but effective way to investigate the triplet decay of nucleosides based on DFT methods, with a quality check at MS-CASPT2 level.

With DFT, all the single point energy calculations were done with $6-311++G(d, p)$ basis set, ${ }^{29}$ using different functionals, including B3LYP $,{ }^{30} \mathrm{X} 3 \mathrm{LYP},{ }^{31}$ and $\omega \mathrm{B} 97 \mathrm{X}-\mathrm{D} .{ }^{32}$ 
Solvent effects were considered with a polarizable continuum model (PCM). ${ }^{13}$ To be consistent with the experiments, water was taken for 6tGua and 6tGuo, while acetonitrile was used for all other species. Gibbs free energy corrections were calculated for the geometry minima. All DFT calculations were carried out with Gaussian $09 .{ }^{33}$

Spin-orbit coupling (SOC) matrix elements between $\mathrm{S}_{0}$ and $\mathrm{T}_{1}$ states were estimated adopting the Breit-Pauli spin-orbit Hamiltonian with effective charge approximation. ${ }^{34}$ For these calculations done with the PySOC program, ${ }^{35}$ the $\mathrm{T}_{1}$ state was recomputed with TDDFT (with Gaussian 09), and an auxiliary wavefunction was built with the Casida’s Ansatz. ${ }^{36}$

Energy-minimum geometry optimizations were first computed at B3LYP/6$311 \mathrm{G}(\mathrm{d}, \mathrm{p})$ level and then updated at $\mathrm{X} 3 \mathrm{LYP} / 6-311++\mathrm{G}(\mathrm{d}, \mathrm{p})$ level. The latter is an extended functional also based on the Lee-Yang-Parr correlation functional, but designed to improve the accuracy of nonbonded interactions, including hydrogen bond and van der Waals interaction. ${ }^{31,37}$ (The addition of sugar substituent in nucleosides should increase the importance of these weak interactions.)

Results from the same MS-CASPT2 level as we used before ${ }^{25}$ were taken as the reference. The active space was composed of 10 electrons in 7 orbitals $\left(1 \mathrm{n}, 4 \pi, 2 \pi^{*}\right)$ using the ANO-RCC-VDZP basis set. $^{38}$ Standard IPEA $(0.25 \mathrm{au})^{39}$ was globally adopted and no level shift ${ }^{40}$ was used. All MS-CASPT2 calculations were done with Molcas $8 .^{28}$

Crossing geometries between the $\mathrm{T}_{1}$ and $\mathrm{S}_{0}$ states were optimized at DFT and MSCASPT2 level, using the penalty function approach up to an energy gap equal or smaller than $0.03 \mathrm{eV}$. This optimization was done with an in-house modified version of the CIOpt program. ${ }^{41}$ Cartesian coordinates of all optimized structures including energy 
minima and crossing points are given in the Electronic Supplementary Information (ESI, Sections S1 to S7).

\subsection{ISC rate analysis}

In our previous work, ${ }^{25}$ the triplet decay rate (or equivalently the $\mathrm{T}_{1} \rightarrow \mathrm{S}_{0}$ ISC rate) of $2 \mathrm{t}$ Thy was determined at the ISC point near the out-of-plane sulfur (op_S) $\mathrm{T}_{1}$ minimum. The rate was calculated following the quasi-Marcus semi-classical formula ${ }^{25,42}$

$$
k_{i s c}=\frac{2 \pi}{\hbar}\left|J_{S O C}\right|^{2} \frac{1}{\sqrt{4 \pi \lambda k_{B} T}} \exp \left(-\frac{\Delta E_{i s c}^{\ddagger}}{k_{B} T}\right),
$$

where $J_{S O C}$ is the SOC at the ISC point; $\lambda$ is the internal reorganization energy computed as the state energy difference of $\mathrm{S}_{0}$ state between the $\mathrm{T}_{1}$ minimum and the $\mathrm{S}_{0}$ minimum; and $\Delta E_{i s c}^{\ddagger}$ is the activation energy corresponding to the $\mathrm{T}_{1}$ state energy difference between the initial op_S minimum and the final crossing point. (For more details, see scheme in the ESI, Section S8.) In the present work, we extended these calculations to several new species. Concerning $\lambda$, in basic Marcus theory involving two diabatic states, the reorganization energy can be equally calculated from either adiabatic state. In our case, however, there is a third diabatic state (the second $\pi \pi^{*}$ state) mixing in $T_{1}$. For this reason, the reorganization energy was calculated using $\mathrm{S}_{0}$, which better fits into Marcus scheme.

Note that this simplified rate model neglects free-energy effects on the reorganization energy and triplet-singlet energy gap, and it also neglects the effect of solvent relaxation to the reorganization energy. Moreover, this equation is strictly valid only if the vibrational frequency promoting the coupling is smaller than $k_{B} T\left(\sim 210 \mathrm{~cm}^{-1}\right.$ at $\left.300 \mathrm{~K}\right)$. Although all these conditions are approximately satisfied for thionucleobases,${ }^{26}$ this rate model is only supposed to deliver a qualitative estimate of the ISC rate. This 
conclusion is especially true if we additionally consider the computational errors in the quantum chemical methods. The crucial problem is the uncertainty in the activation energy, which goes into the exponential function, amplifying the error. Having all these limitations in mind, our initial expectation was not to obtain more than qualitative ratios between rates. Nonetheless, the model revealed to be exceptionally accurate, delivering rates in semi-quantitative agreement with the experiments, as we present later.

\section{Mechanism of Enhanced Triplet Decay by Sugar Group}

\subsection{Calculation of 2tThy and confirmation of DFT performance}

The triplet decay of 2tThy has been calculated at MS-CASPT2/ANO-RCC-VDZP level in previous work, ${ }^{25}$ and the rate-determining step of triplet decay is the intersystem crossing from op_S minimum through the ISC geometry. The primary molecular motion driving the process is the out-of-plane vibration of the sulfur atom along the angle $\beta=\angle \mathrm{C} 5 \mathrm{C} 2 \mathrm{~S}$ (Figure 1). ${ }^{25}$ Here, we re-optimized the geometry of $2 \mathrm{tThy}$ with DFT methods. The geometries from B3LYP/6-311G(d,p), X3LYP/6-311++G(d,p), and MSCASPT2/ANO-RCC-VDZP are nearly the same, with the variation of the $\beta$ angle being less than 2 degrees. Then, we calculated the energy profiles with linear interpolation of internal coordinates (LIIC) with different functionals and compared them with the one from the MS-CASPT2 method. This comparison is shown in Figure 2.

The nearly same energy profiles confirm the satisfactory performance of DFT. These results also imply that the performance is not particularly sensitive to the choice of functionals. We recall that the two curves are both from ground-state DFT calculations (not TDDFT), which explains the robustness of the results. 


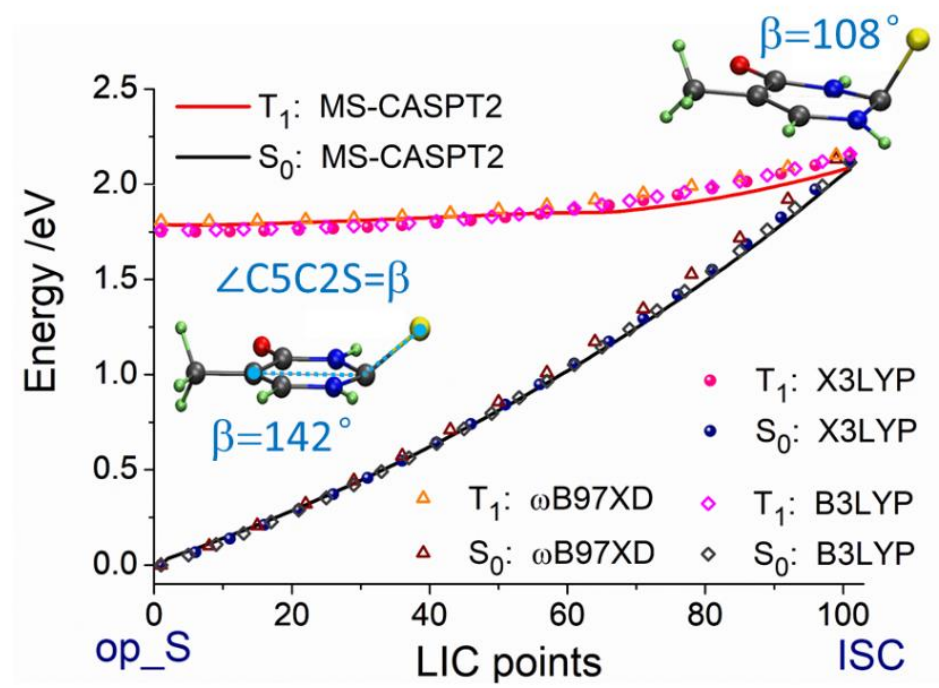

Figure 2. Energy profiles of the $T_{1}$ and $S_{0}$ states connecting the op_S minimum and the ISC point: geometries optimized and energy calculated at MS-CASPT2 (solid lines). For the DFT calculations (points), geometries from X3LYP level are used.

\subsection{Choice of thionucleosides conformer}

When we consider the thionucleosides, the first thing to ponder is the conformational isomers because of the added sugar group. Rotation of the single-bond connecting nucleobase and the sugar, as well as rotations within the sugar group, generates different conformers. The conformers of thymidine and uridine analogs have been investigated by other groups, and more than 5,000 geometries could be optimized. ${ }^{43,44}$ It is out of the scope of this work to present a comprehensive conformational analysis, and for our purposes, the rotation of the connecting single bond is the most relevant parameter determining the nucleoside geometry. To keep it manageable, we consider the conformational effect in the two lowest energy conformers, which are usually named anti and syn (Figure 3). In these two conformers, their rotations are opposite as the $\sim 180^{\circ}$ difference of the dihedral angle $\chi$ tells; and in both conformers the two ring planes are nearly perpendicular to each other, minimizing the intramolecular repulsion 
between them. In the anti conformer, the sugar ring tends to approach the hydrogen atom in position 6 , while in the syn conformer it is closer to the larger sulfur atom in position 2, making the repulsion in the former weaker than in the latter, and the anti conformer more stable.

(a) anti

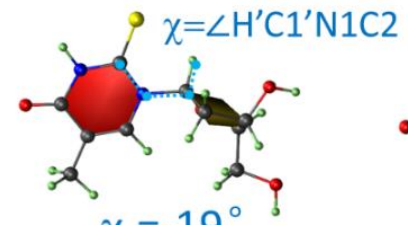

$\chi=-19^{\circ}$ (b) syn

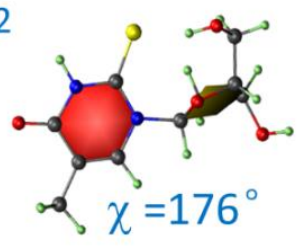

$\Delta \mathrm{G}_{\mathrm{s} 0}=0.0 \mathrm{eV} \quad \Delta \mathrm{G}_{\mathrm{s} 0}=0.19 \mathrm{eV}$

Figure 3. The anti (a) and syn (b) conformers of 2 tThd optimized in the ground state at $\mathrm{X} 3 \mathrm{LYP} / 6-311++\mathrm{G}(\mathrm{d}, \mathrm{p})$ level. The Gibbs free energy variation $\Delta \mathrm{G}_{\mathrm{S} 0}$ of ground state is taken with the anti conformer as the reference. The definition and values of the dihedral angle $\chi$ are shown in blue.

We optimized the ground state minima of both anti and syn conformers of 2 tThd and calculated their Gibbs free energy. Their structures are shown in Figure 3, and the free energy difference between anti and syn conformers $-\Delta \mathrm{G}_{\mathrm{S} 0}=0.19 \mathrm{eV}$, corresponding to a Boltzmann factor smaller than $0.1 \%$ - grants that only the anti conformer is populated in the ground state at room temperature. This situation is distinct from Thd, which is well known for featuring a small but relevant syn population. ${ }^{43}$ In fact, if we repeat the same estimates for Thd, we find out that $\Delta \mathrm{G}_{\mathrm{S} 0}=0.07 \mathrm{eV}$, corresponding to a Boltzmann factor of $7 \%$ under the same conditions. The difference between 2 tThd and Thd in this regard is the larger size of the sulfur compared to the oxygen atom, which increases the intramolecular repulsion and, thus, the difference between anti and syn conformers. This repulsion effect also slightly changes the 
chromophore geometries. With the sugar group, the sulfur atom is deflected toward N3, as the $\beta$ angle reduces from $179.4^{\circ}$ in Thy to $176.6^{\circ}$ in anti and $174.9^{\circ}$ in syn $2 \mathrm{tThd}\left(\mathrm{S}_{0}\right.$ minimum).

\subsection{Effects of deoxyribose/ribose in $2 \mathrm{tThd} / 2 \mathrm{tThr}$}

The $\mathrm{T}_{1}$ state of 2 tThy features two ${ }^{3} \pi \pi^{*}$ minima. ${ }^{25}$ Beyond the op_S minimum discussed above, there is another one with boat $\mathrm{B}_{3.6}$ conformation. In the discussion of Section 3.1, we have neglected this second minimum because the ISC from op_S is the rate-determining step. ${ }^{25}$ In the case of $2 \mathrm{tThd}$ and $2 \mathrm{tThr}$, the relevance of the op_S minimum should be even bigger because of the repulsion between the sugar group and the sulfur atom, which should destabilize the $\mathrm{B}_{3,6}$ minimum. This assumption has been confirmed by computing the energy gap between the two minima for $2 \mathrm{tTh}$ conformers at different levels (DFT, CC2, and CASPT2). Only the geometries from anti conformer were considered because they are essential for the final rate, as explained in Subsection 3.3.3. For all levels, the results (see ESI, Section $S 9$ ) show that the $T_{1}$ energy of the $B_{3,6}$ minimum relative to the energy of the op_S minimum of $2 \mathrm{tThd}$ is larger than this same energy gap in 2 tThy. Thus, we can proceed exclusively focusing on the op-S minimum.

The overall topography of the $\mathrm{T}_{1}$ state of $2 \mathrm{tThd}$ is similar to that of $2 \mathrm{tThy}$, but the addition of the deoxyribose enabling anti and syn conformers creates new possible minima. Due to its mirror symmetry, for 2tThy it makes no difference whether the sulfur is displaced up or down the ring plane. Nevertheless, for $2 \mathrm{tThd} / 2 \mathrm{tThr}$ these two possibilities correspond to distinct minima, and we name them "up" and "down" depending on how the sulfur is displaced out of the plane, as shown in Figure 4. Finally, we should have four minima arising from the two rotation (anti/syn) options and two S-displacement (up/down) options. 


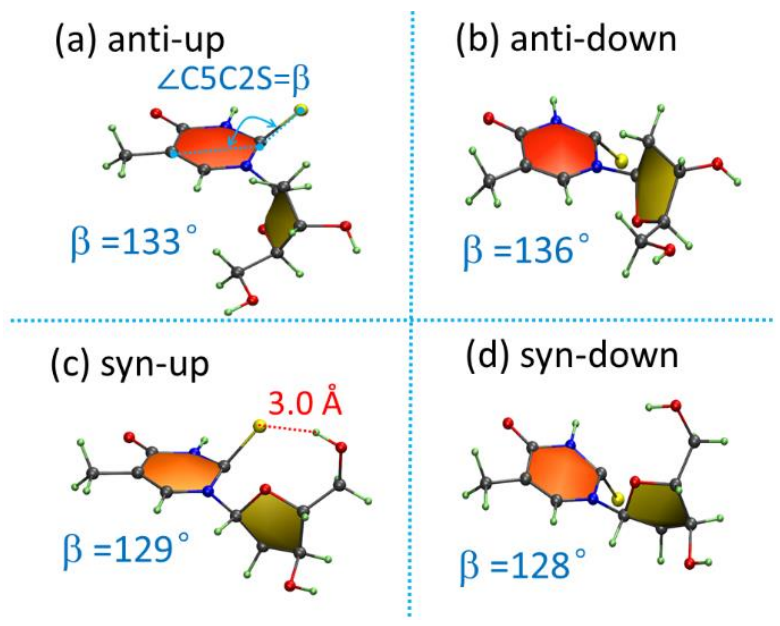

Figure 4. Four different optimized $\mathrm{T}_{1}$ minima of $2 \mathrm{tThd} \beta=\angle \mathrm{C} 5-\mathrm{C} 2-\mathrm{S}$ angle is given in degrees.

Following these conformational guidelines, we optimized the $\mathrm{T}_{1}$ minima of $2 \mathrm{tTh}$ and $2 \mathrm{tThr}$ with DFT in the same way as $2 \mathrm{tThy}$ and obtained the four $\mathrm{T}_{1}$ minima: antiup/down and sys-up/down. Their geometries are shown in Figure 4 and Figure 5. The $\beta$ angle, which is taken to indicate the out of plane motion of sulfur atom, is nearly the same for $2 \mathrm{tThd}$ and $2 \mathrm{tThr}$.

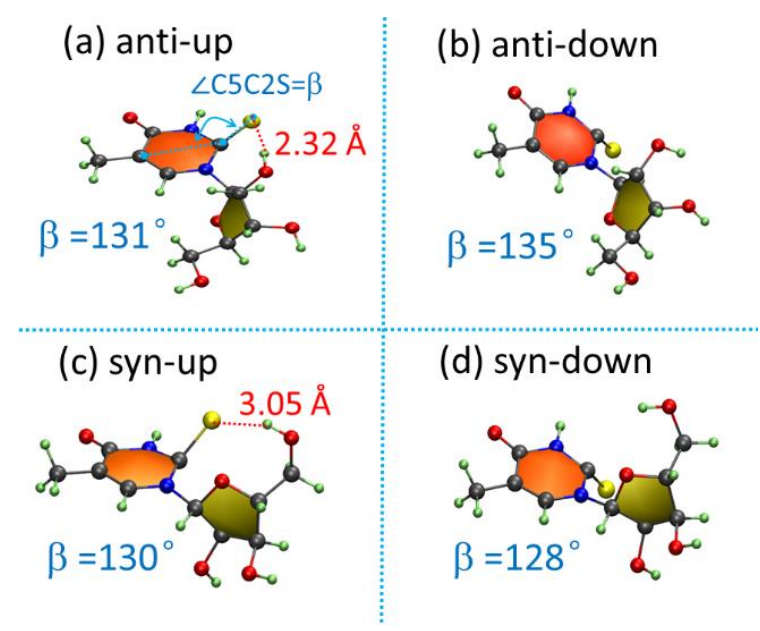

Figure 5. Four different optimized $\mathrm{T}_{1}$ minima of $2 \mathrm{tThr}$, with the same labels as in Figure 4. 
For all four op-S minima of $2 \mathrm{tThd}$, the four of $2 \mathrm{tThr}$, and the op_S minimum of $2 \mathrm{tThy}$, geometric, energetic, and coupling parameters are collected in Table 1. These data will be discussed in the next subsections and used to compute the ISC rate discussed in the Subsection 3.3.3.

Table 1. Calculated parameters of $2 \mathrm{tThy}, 2 \mathrm{tThd}$, and $2 \mathrm{tThr}$ based on the op_S minima for the triplet decay. $\beta$ : $\angle \mathrm{C} 5-\mathrm{C} 2-\mathrm{S}$ angle; $\mathrm{d}(\mathrm{S}-\mathrm{OH})$ : shortest distance between sulfur and the hydrogen on sugar group at the ISC point; $J_{S O C}$ : spin-orbit coupling between $\mathrm{T}_{1} / \mathrm{S}_{0}$ states at the ISC point; $\lambda$ : reorganization energy; $\Delta E_{i s c}^{\ddagger}$ activation energy for ISC.

\begin{tabular}{|c|c|c|c|c|c|c|c|c|c|}
\hline \multirow{2}{*}{} & \multirow{2}{*}{2 2tThy } & \multicolumn{4}{|c|}{ 2tThd } & \multicolumn{4}{c|}{ 2tThr } \\
\cline { 3 - 11 } & & anti-up & anti-down & syn-up & syn-down & anti-up & anti-down & syn-up & syn-down \\
\hline$\beta\left(\mathrm{T}_{1} \mathrm{~min}\right) /^{\circ}$ & 142 & 133 & 136 & 129 & 128 & 131 & 135 & 130 & 128 \\
\hline$\beta(\mathrm{ISC}) /^{\circ}$ & 108 & 107 & 106 & 117 & 108 & 105 & 106 & 116 & 105 \\
\hline $\mathrm{d}(\mathrm{S}-\mathrm{OH}) / \AA$ & & 6.3 & 6.4 & 2.9 & 5.9 & 2.5 & 5.2 & 2.8 & 4.1 \\
\hline$\Delta E_{i s c}^{\ddagger} / \mathrm{eV}$ & 0.40 & 0.33 & 0.32 & 0.23 & 0.28 & 0.32 & 0.33 & 0.24 & 0.30 \\
\hline$J_{S O C} / \mathrm{cm}^{-1}$ & 74 & 76 & 74 & 93 & 87 & 73 & 74 & 93 & 91 \\
\hline$\lambda / \mathrm{eV}$ & 1.24 & 1.37 & 1.30 & 1.51 & 1.55 & 1.49 & 1.28 & 1.51 & 1.54 \\
\hline
\end{tabular}

\subsubsection{Effect of the sugar group on geometry and activation energy}

We first focus on the geometry change along the $T_{1}$ pathway between the minimum and the ISC point for 2 tThy. As shown in Figure 2, the $\beta$ angle, the main internal coordinate in this pathway, reduces from $142^{\circ}$ to $108^{\circ}$, an angular distance of $34^{\circ}$. According to Figure 4, Figure 5, and Table 1, this angular distance is always smaller in 2tThd/2tThr (between $12^{\circ}$ and $30^{\circ}$ depending on the minimum). Thus, the ISC reaction pathway is shorter in the thionucleoside than in the thionucleobase. Consequently, the activation energy $\Delta E_{i s c}^{\ddagger}$ of the thionucleobases is $0.07-0.17 \mathrm{eV}$ smaller than that of $2 \mathrm{tThy}$. All rest being equal, Eq. (1) predicts that a $\delta \varepsilon$ reduction in the activation energy should lead to a rise of about $\exp \left(\delta \varepsilon / k_{B} T\right)$ in the rate. Thus, at room temperature, this activation energy reduction corresponds to 15 to 1000 times of rate raise. 
For anti (either up or down) and syn-down conformers, the shorter angular distance is due to the smaller $\beta$ at the $T_{1}$ minimum. This difference arises from the repulsion interaction between the sugar group and the sulfur atom, pushing the sulfur to a larger out-of-plane displacement. For the syn-down conformer, the $\beta$ angle at the minimum and the angular distance to the crossing are smaller than for the anti conformers, reinforcing this interpretation because the repulsion interaction in the syn conformers strains the structures even more. At the ISC geometries of these conformers, the sulfur atom lies farther away from the sugar group, and this repulsion effect is much smaller, so the $\beta$ angle in 2 tThd and $2 \mathrm{tThr}$ are nearly the same as in $2 \mathrm{tThy}$. The difference in the strength of the repulsion at the $\mathrm{T}_{1}$ minimum and the ISC point explains the activation energy reduction. Along the reaction path, the $\mathrm{T}_{1}$ state energy is more affected by the repulsion effect near the minimum than near the ISC point. Thus, the activation energy $\Delta E_{i s c}^{\ddagger}$ - the $\mathrm{T}_{1}$ energy difference between these two points - is reduced.

This explanation is not enough for the syn-up conformers, where the ISC geometry and final activation energy are distinct from those of the other conformers. The $\beta$ angle at the $\mathrm{T}_{1}$ minima of syn-up and syn-down conformers are nearly the same, $12^{\circ}$ smaller than that of 2 tThy, indicating the repulsion effect also exists for syn-up conformer. However, the $\beta$ angle at the ISC point of the syn-up conformer is about $10^{\circ}$ larger than the ones of the other conformers and the one of 2tThy. As a result, the angular distance of the ISC reaction path of syn-up conformers is the shortest, and the activation energy is the smallest. The strain in the syn-up ISC point is due to an intramolecular hydrogen bond occurring in this conformer. Although the $\mathrm{S}-\mathrm{H}$ distance in the syn-up $\mathrm{T}_{1}$ minimum is $3.0 \AA$ for 2 tThd, it decreases to $2.86 \AA$ at the ISC point, which is in the range of 
typical hydrogen bonds with a sulfur acceptor. ${ }^{45}$ As obtained in previous work, ${ }^{25}$ the two singly-occupied MOs of the $\mathrm{T}_{1}$ state at the op_S minimum are the bond and antibond orbitals along the $\mathrm{C}=\mathrm{S} \pi$ bond as shown in Figure 6; and the anti-bond orbital has a larger spatial extension to form a hydrogen bond. Then, the intramolecular hydrogen bond stabilizes the $\mathrm{T}_{1}$ state more than the $\mathrm{S}_{0}$ state, decreasing their energy gap. Finally, this interaction leads to a bigger $\beta$ angle at the crossing point, a shorter angular distance of the ISC reaction, and smaller activation energy $\Delta E_{i s c}^{\ddagger}$.

According to the $d(\mathrm{~S}-\mathrm{OH})$ in Table 1 and the geometry in Figure 5(a), the anti-up conformer of $2 \mathrm{tThr}$ also seems to form an intramolecular $\mathrm{H}$-bond, but the angular distance and activation energy are nearly the same as the ones of 2 tThd. This apparent contradiction arises from two reasons: first, the N1-C2-S-H dihedral angle is only $38^{\circ}$, which is not suitable for S-HO hydrogen bond formation, which is favored ${ }^{46}$ at $90^{\circ}$ (for comparison, this angle is $54^{\circ}$ for syn-up conformer). Second — and most important - for this conformer, the S-H distance at $\mathrm{T}_{1}$ minima is even shorter than at the ISC point. Then, the stabilization effect acts along the whole pathway and does not decrease the activation energy.
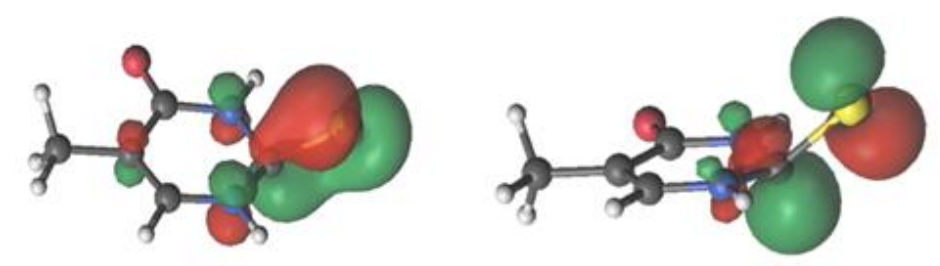

Figure 6. Singly-occupied orbitals of the $\mathrm{T}_{1}$ state of 2 thy from CASPT2 calculation at the op_S minimum. ${ }^{25}$

To summarize, for both anti and the syn-down conformers, the reduction in the activation energy is due to the sugar-sulfur repulsion, while for the syn-up conformer, 
it is additionally due to the intramolecular hydrogen bond stabilizing the $T_{1}$ state energy at the ISC point.

\subsubsection{Effect of sugar group on SOC and reorganization energy}

Compared to the SOC of $2 \mathrm{tThy}$ at the crossing geometry $\left(74 \mathrm{~cm}^{-1}\right)$, the SOC of $2 \mathrm{tThd}$ and $2 \mathrm{tThr}$ for anti conformers are nearly the same, while the ones for syn conformers $\left(93 / 87 / 93 / 91 \mathrm{~cm}^{-1}\right)$ are somewhat larger. Thus, the stronger repulsion effect seems to increase the SOC. However, as the number tells, the largest SOC among $2 \mathrm{tThd}$ isomers is 1.3 times that of 2 tThy. Either directly with the Fermi-golden rule or the quasiMarcus limit Eq. (1), we know that the triplet decay rate is proportional to the square of SOC. Then, with all rest being equal, the largest SOC here could only lead to a maximum rate enhancement of about 1.7 times that of 2 tThy, while the experimental results showed an over 10 times increase of the triplet decay rate. As a conclusion, the SOC alone cannot be the core reason for the enhanced decay rate.

As shown in Table 1, the calculated internal reorganization energy $\lambda$ of four $T_{1}$ minima of $2 \mathrm{tThd}(1.37 / 1.30 / 1.51 / 1.55 \mathrm{eV})$ is only slightly larger than that of $2 \mathrm{tThy}$ $(1.24 \mathrm{eV})$. According to Eq. (1), $\lambda$ affects the rate with a $\lambda^{-1 / 2}$ relationship. Thus, its increase from $1.24 \mathrm{eV}$ to the largest value, $1.55 \mathrm{eV}$, would change the rate by a factor 0.89. Therefore, the effect from the reorganization energy tends to be opposite to the experimental trend, but it is small enough to be neglected.

Comparing to the effect of activation energy, the changes of SOC and $\lambda$ are both tiny, and they tend to cancel out each other. Therefore, the change of activation energy is clearly the dominant factor for the enhanced triplet decay rate of the thionucleosides. 


\subsubsection{Calculated triplet decay rate and comparison to experiment}

Having computed the three variables in Eq. (1) $-J_{\mathrm{SOC}}$, reorganization energy $\lambda$, and activation energy $\Delta E_{i s c}^{\ddagger}$ (Table 1), we calculated the final triplet decay rate $k_{i s c}$ for the four $T_{1}$ minima. The results are shown in Table 2.

Table 2. Quantities characterizing the triplet decay of 2tThy, 2tThd, and $2 \mathrm{tThr}$. Theor. $k_{i s c}$ : triplet decay rate calculated with Eq. (1); Expt. $k_{i s c}$ : triplet decay rate experimentally measured $;^{13} \Delta G\left(\mathrm{~S}_{0}\right)$ : Gibbs free energy difference in $\mathrm{S}_{0} ; \Delta G\left(\mathrm{~T}_{1}\right)$ : Gibbs free energy difference in $\mathrm{T}_{1}$.

\begin{tabular}{|c|c|c|c|c|c|}
\hline & & $\begin{array}{l}\text { Theor. } \\
k_{i s c} \\
/ 10^{6} \mathrm{~s}^{-1}\end{array}$ & $\begin{array}{l}\text { Expt. } k_{i s c} \\
/ 10^{6} \mathrm{~s}^{-1}\end{array}$ & $\begin{array}{l}\Delta G\left(\mathrm{~S}_{0}\right) \\
/ \mathrm{eV}\end{array}$ & $\begin{array}{l}\Delta G\left(\mathrm{~T}_{1}\right) \\
/ \mathrm{eV}\end{array}$ \\
\hline \multicolumn{2}{|l|}{ 2tThy } & 0.24 & $\begin{array}{c}0.1111 \pm 0.0004 \\
(0.37 \pm 0.04)^{47}\end{array}$ & 0.00 & 0.00 \\
\hline \multirow[t]{4}{*}{ 2tThd } & anti-up & 3.6 & \multirow[t]{4}{*}{$1.6 \pm 0.2$} & 0.00 & 0.03 \\
\hline & anti-down & 5.2 & & 0.00 & 0.00 \\
\hline & syn-up & 250 & & 0.19 & 0.04 \\
\hline & syn-down & 31 & & 0.19 & 0.11 \\
\hline \multirow[t]{4}{*}{2 tThr } & anti-up & 4.7 & \multirow[t]{4}{*}{$2.5 \pm 0.2$} & 0.00 & 0.17 \\
\hline & anti-down & 3.6 & & 0.00 & 0.00 \\
\hline & syn-up & 170 & & 0.25 & 0.14 \\
\hline & syn-down & 16 & & 0.25 & 0.23 \\
\hline
\end{tabular}

For $2 \mathrm{tThy}$, the calculated rate is close to the experimental one within a value two. Moreover, the theoretical values lie between two independent experimental estimates. $^{13,47}$ Given all computational and theoretical limitations discussed in Subsection 2.2, these are indeed reassuring facts vouching for the validity of the method.

For $2 \mathrm{tThd}$ and $2 \mathrm{tThr}$, we need to consider the relative population of their four conformers at the $T_{1}$ minimum. To do so, we should start from the initial ground-state $\left(\mathrm{S}_{0}\right)$ distribution. As we concluded in Subsection 3.2, only anti conformers of these thionucleosides are initially populated. Then, after excitation and fast relaxation to $\mathrm{T}_{1}$ 
state, this character remains, initially generating equal amounts of anti-up and antidown conformers approximately. Due to the $\mu$ s-scale of the decay to the ground state, ${ }^{13}$ we need to consider the transitions between the four conformers. For the transition between anti-up and anti-down, restricted optimization of the $T_{1}$ state with a planar sulfur atom $(\beta=0)$ shows that the activation energy for this transition is larger than 0.4 $\mathrm{eV}$. Therefore, this transition can be discarded.

Then, we just need to consider the transition from anti to syn conformers. Nevertheless, this determination revealed to be out of our current capabilities. First, computing an accurate energy barrier for the large volume-changing anti $\rightarrow$ syn transition requires explicit treatment of the solvent, beyond the continuous field adopted here. Second, even if we got this barrier, the anti $\rightarrow$ syn transition is driven by multiple torsional motions, with low frequency, high amplitude, and poor description via normal modes. A simple rate treatment like that of Eq. (1) would not deliver reliable results. In fact, reliable theoretical methods to compute rates for such challenging problem is still under development. ${ }^{48}$ Given these methodological limitations, we decided to invert the analysis and use the comparison between experiment and theory to tell about the mechanism. In this way, the excellent agreement between theoretical and computational rates shown Table 2 for anti conformers is a strong indication that the anti $\rightarrow$ syn transition does not take place.

The final rate results are given in Table 3 with the simple average of the anti conformers. Given the strong approximations involved in the computation of the rates via Eq. (1) and in the underlying electronic structure calculations, the semiquantitative agreement between theoretical and experimental results is remarkable. Although the theoretical rates are systematically bigger than the experimental values ${ }^{13}$ by a factor 
from 1.7 to 3 , the ratios between the rates for thionucleosides and thionucleobases captures the primary effect of triplet decay enhancement induced by the sugar group.

Table 3. Theoretical (Eq. (1)) and experimental ${ }^{13}$ rates and rate ratios for the triplet decay of $2 \mathrm{tThy}, 2 \mathrm{tThd}$, and $2 \mathrm{tThr}$ in acetonitrile. Theoretical results for the arithmetic average of anti conformers.

\begin{tabular}{|c|c|c|c|c|}
\hline & \multicolumn{2}{|c|}{ Rate $/ 10^{6} \mathrm{~s}^{-1}$} & \multicolumn{2}{|c|}{ Rate ratio } \\
\hline & Theory & Expt. & Theory & Expt. \\
\hline 2tThy & 0.24 & $\begin{array}{c}0.1111 \pm 0.0004 \\
(0.37 \pm 0.04)^{47}\end{array}$ & 1 & 1 \\
\hline 2tThd (anti $i_{\mathrm{av}}$ ) & 4.4 & $1.6 \pm 0.2$ & 18 & $14 \pm 2$ \\
\hline $2 \mathrm{tThr}\left(\right.$ anti $\left.\mathrm{i}_{\mathrm{av}}\right)$ & 4.2 & $2.5 \pm 0.2$ & 17 & $23 \pm 2$ \\
\hline
\end{tabular}

\subsection{Effects of deoxyribose in 6tGuo}

Recalling the discussion in the Introduction, the experimental rate for the triplet decay of $2 \mathrm{tThd}$ is much larger than that of $2 \mathrm{tThy}$ by a factor $14 .{ }^{13}$ Nevertheless, the experimental rate for the triplet decay of 6tGuo is much closer to that of 6tGua, just twice larger. ${ }^{14}$ With the theoretical tools used in the previous section, we are in a position to explain this difference between the photophysics of the thiopyrimidines and thiopurines.

We know from previous studies that 6tGua has similar triplet decay pathway as 2 tThy, with the sulfur out-of-plane motion being essential, and the lowest $T_{1}$ minima also bearing an op_S structure..$^{22,25,49} 6 \mathrm{tGuo}$, characterized by the glycosylation of 6tGua in the N9 position, should share similar triplet state topography. The $\mathrm{T}_{1}$ minima of 6tGua and 6tGuo, shown in Figure 7, were optimized at the X3LYP/6-311++g(d,p) level. For 6tGuo, the direction of sulfur out-of-plane is not an issue because of the considerable distance separating this atom from the sugar group. Therefore, just antiup and syn-up conformers are presented. For the same reason, the N9-glycosylation 
does not significantly change these minima from 6tGua to 6tGuo, which share nearly the same $\beta$ angle.

(a) 6tGua

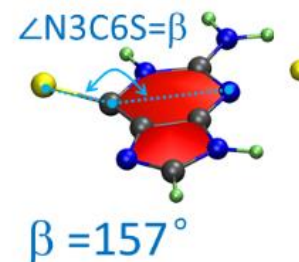

(b) 6tGuo: anti

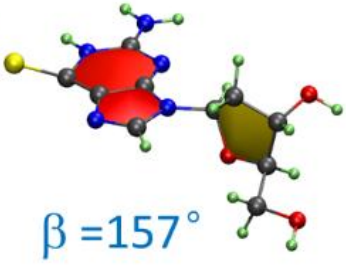

(c) 6tGuo: syn

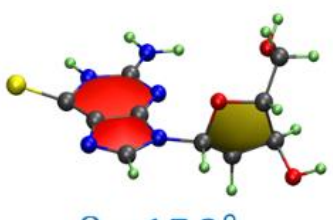

$\beta=156^{\circ}$

Figure 7. Optimized $\mathrm{T}_{1}$ minima of (a) $6 \mathrm{tGua}$ and of (b) anti and (c) syn conformers of $6 \mathrm{tGuo}$.

Table 4. Properties of 6tGua and 6tGuo for the triplet decay. The labels are the same as in Table 1 and Table 2. The rate ratio takes the rate of $6 \mathrm{tGua}$ as the reference.

\begin{tabular}{|l|c|c|c|}
\hline & 6tGua & 6tGuo \\
\cline { 3 - 4 } & & anti-up & syn-up \\
\hline$\Delta E_{i s c}^{\ddagger} / \mathrm{eV}$ & 0.364 & 0.358 & 0.369 \\
\hline$J_{S O C} / \mathrm{cm}^{-1}$ & 44 & 44 & 44 \\
\hline$\lambda / \mathrm{eV}$ & 0.50 & 0.52 & 0.49 \\
\hline$k_{\text {isc }}$ theory $/ 10^{6} \mathrm{~s}^{-1}$ & 0.54 & 0.67 & 0.45 \\
\hline $\mathrm{k}_{\text {isc }}$ expt. $/ 10^{6} \mathrm{~s}^{-1}$ & $0.70 \pm 0.09^{\mathrm{a}}$ & $1.40 \pm 0.02^{\mathrm{a}}$ \\
\hline Ratio theory & 1 & 1.24 & 0.83 \\
\hline Ratio expt. & 1 & $2.0 \pm 0.3^{\mathrm{a}}$ \\
\hline$\Delta \mathrm{G}\left(\mathrm{S}_{0}\right) / \mathrm{eV}$ & 0.0 & \multicolumn{3}{|c|}{0.0} & 0.06 \\
\hline
\end{tabular}

${ }^{\mathrm{a}}$ In PBS pH 7.4 solution with saturated $\mathrm{N}_{2}$, from Ref. ${ }^{14}$

We also calculated the parameters and the triplet decay rates using Eq. (1), and the results are collected in Table 4 . In the ground state, the anti conformer is $0.06 \mathrm{eV}$ more stable than syn. Thus, at room temperature, we may expect a non-negligible syn population of about $10 \%$. As in the case of $2 \mathrm{tThd}$, we cannot estimate the anti and syn populations in the $T_{1}$ state. Despite that, whatever this composition is, the rate values are in a good agreement with the experiment. Like in the experiment, the calculated rate also shows that the triplet decay rate of 6tGuo is not significantly enhanced compared 
to that of $6 \mathrm{tGua}$. The $6 \mathrm{tGuo} / 6 \mathrm{tGua}$ rate ratio is 1.24 for anti and 0.83 for syn, comparing well to the experimental value 2.0.

\section{Modulating the triplet decay rate}

From the theoretical results for $2 \mathrm{tThd} / 2 \mathrm{tThy}$ and $6 \mathrm{tGuo} / 6 \mathrm{tGua}$, we learned that, first, the interaction between the sulfur and the sugar group changes the $T_{1}$ energy unevenly along the pathway connecting the $\mathrm{T}_{1}$ minimum and the $\mathrm{T}_{1} / \mathrm{S}_{0}$ crossing point. This effect leads to a decrease in the ISC activation energy and increase of the triplet decay rate. Second, this effect on the rate is reduced by widening the distance between the sulfur atom and the sugar group. Moreover, we also noticed that for $2 \mathrm{tThd}$ and $2 \mathrm{tThr}$ the rates for syn conformers might be much larger than those for anti conformers (Table 2).

These mechanistic results provide us with information to propose new thionucleoside derivatives specifically designed to control the triplet decay rate, with the ambition that they would be practical and inspire experimentalists in different fields. For instance, to enhance even more the triplet decay we could follow two strategies: (1) stabilizing of the syn conformer; (2) increasing the rate of the anti conformer by rising the repulsion with the sulfur atom.

Concerning the first strategy, for $2 \mathrm{tThd}$, the anti conformers are more stable because the sugar group is farther away from the large sulfur atom at position 2 and closer to the small hydrogen atom at position 6 , while for syn conformers it is opposite. If, therefore, we replace that hydrogen with a larger group, then the state energy of anti conformers would increase, and syn could be more stable. Following this hypothesis, if, for instance, we replace the hydrogen atom at position 6 by a methyl group (6methyl-2-thiothymindine, 6m-2tThd), the syn conformer becomes only $0.07 \mathrm{eV}$ less 
stable than the anti (Figure 8), which should substantially increase its population and, consequently, the triplet decay rate.

(a) anti

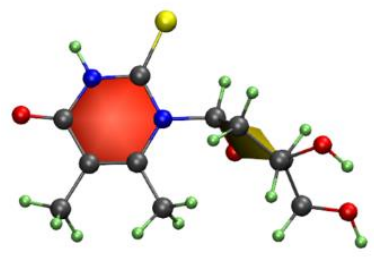

$\Delta \mathrm{G}_{\mathrm{S} 0}=0.0 \mathrm{eV}$ (b) syn

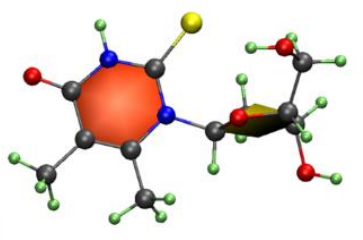

$\Delta \mathrm{G}_{\mathrm{s} 0}=0.07 \mathrm{eV}$

Figure 8. Anti (a) and syn (b) conformers of $6 \mathrm{~m}-2 \mathrm{tTh}$ optimized at the ground state. The labels are the same as in Figure 3.

The second strategy aims at increasing the triplet decay rate of anti conformer itself. As discussed above, the decay enhancement mainly comes from the intermolecular repulsion between the sugar group and the sulfur atom at $\mathrm{T}_{1}$ minimum, causing a reduction of activation energy. Therefore, if we modify the molecule to increase this repulsion effect in the anti conformer, the decay rate should also be additionally enhanced.

At the anti conformer of 2 tThd, one of the leading sources of repulsion is the interaction between the sulfur atom and the hydrogen at position 1' of the sugar group. Then, a natural trial is to replace this hydrogen atom with a larger atom or group. If, for instance, a methyl group replaces it, the modified molecule - 1'-methyl-2thiothymidine (1'm-2tThd) - still has the anti conformer as the most stable, $0.23 \mathrm{eV}$ lower than the syn conformer. 

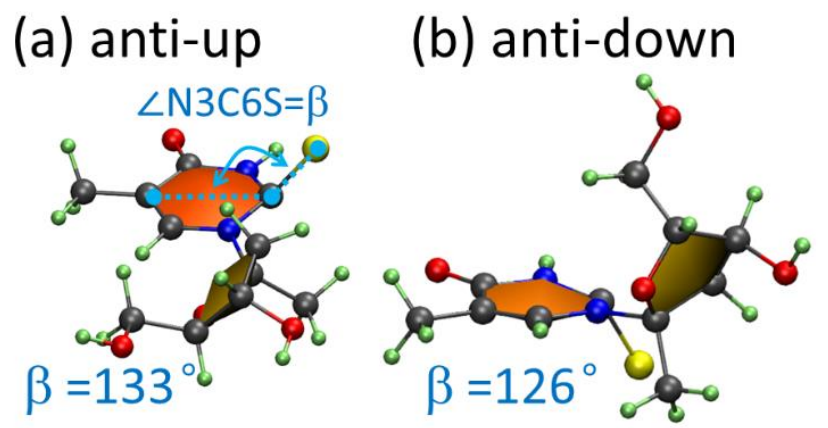

Figure 9. The optimized $\mathrm{T}_{1}$ minima (a) anti-up and (b) anti-down of anti conformers of 1'm2tThd.

Starting from the anti ground-state minimum geometry, we optimized at $\mathrm{T}_{1}$ state, and obtained $2 \mathrm{~T}_{1}$ minima, anti-up and anti-down, as shown in Figure 9. Then the parameters and final decay rate were calculated and collected in Table 5. It shows that although $\lambda$ and $J_{\text {soc }}$ are nearly the same as those of 2 tThd in Table 1 , the activation energy $\Delta E_{i s c}^{\ddagger}$ is smaller, and the rates are larger. The average rate from the two anti conformers of 1 'm-2tThd is 243 times larger than the rate of $2 \mathrm{tThy}$, a substantial enhancement in comparison to the original molecule $2 \mathrm{tThd}$, which is only 14 times larger.

Table 5. Properties of 1 'm-2tThd for the triplet decay. The labels are the same as in Table 1 and Table 2. The rate ratio takes the rate of 2 tThy as the reference.

\begin{tabular}{|l|c|c|}
\hline & anti-up & anti-down \\
\hline$\Delta E_{i s c}^{\ddagger} / \mathrm{eV}$ & 0.31 & 0.25 \\
\hline$J_{S O C} / \mathrm{cm}^{-1}$ & 74 & 89 \\
\hline$\lambda / \mathrm{eV}$ & 1.26 & 1.40 \\
\hline$k_{\text {isc }}$ theory $/ 10^{6} \mathrm{~s}^{-1}$ & 7.8 & 110 \\
\hline Ratio to 2tThy & 32 & 453 \\
\hline Average Ratio & \multicolumn{2}{|c|}{243} \\
\hline
\end{tabular}

Since we already know how sugar group enhances the triplet decay, we could also propose strategies to decrease the rate, namely introducing the opposite effects to 
increase the activation energy. This could be achieved by (1) adding a group that can generate more repulsion at ISC point than at the $\mathrm{T}_{1}$ minimum, or (2) promoting the formation of a hydrogen bond at the $\mathrm{T}_{1}$ minimum, but not at the ISC point.

\section{Conclusion}

In this work, we have determined the underlying molecular mechanism that explains why triplet decay rates of thionucleosides are enhanced compared to that of thionucleobases. Considering that these substances have recently acquired vast importance for diverse types of clinical treatments, this question is not only relevant for fundamental physical chemistry, but also for potential medicinal applications.

Based on the mechanism of triplet decay of thionucleobases found in our previous work, here we used density functional theory to characterize the topography of the $T_{1}$ and $\mathrm{S}_{0}$ states (both ground states of their multiplicities) and kinetic modeling to compute the triplet decay rate. These methods were used to simulate the most significant conformers of a series of thio-pyrimidines (2tThy/2tThd/2tThr) and thiopurines (6tGua/6tGuo).

The reduction of the activation energy for $\mathrm{T}_{1} / \mathrm{S}_{0}$ intersystem crossing was finally determined to be the main reason for the enhanced decay rate. It is caused by the intramolecular repulsion between the sulfur atom and the sugar group, which changes the $T_{1}$ state energy unevenly along the reaction path connecting the $T_{1}$ minima to the intersystem $T_{1} / S_{0}$ crossing point. The reduction of activation energy is also reinforced in specific conformers by the formation of an intramolecular hydrogen bond between the sugar group and the aromatic chromophore.

This molecular mechanism, as well as the result that anti and syn conformers of 2tThd have strikingly different decay rates, provides some general guidelines for 
molecular design to modulate the triplet decay rate. We have tested them onto a couple of new thionucleoside derivatives, computationally confirming the validity of this strategy.

This work was built upon a detailed investigation of the triplet decay pathway beyond the static analysis, including the calculation of reaction rates. It has allowed us to unveil the molecular mechanism of a crucial photophysical step in the reactivity of thionucleoside derivatives. Our model has been validated by strict comparison to experimental data available in the literature, and its excellent performance has led us to propose heuristic directives to control the reaction rates. We expect that these directives may open new research possibilities in the experimental domain, through the design and synthesis of new compounds with tailored photophysical responses.

\section{Electronic Supplementary Information (ESI)}

Electronic supplementary information (ESI) available: Cartesian coordinates of optimized structures; detailed information about Eq. (1); comparison between $\mathrm{T}_{1}$ minima. See DOI: 10.1039/x0xx00000x

\section{Acknowledgments}

The authors thank the support of the Excellence Initiative of Aix-Marseille University (A*MIDEX) and the project Equip@Meso (ANR-10-EQPX-29-01), both funded by the French Government "Investissements d'Avenir" program. MB also acknowledges funding the WSPLIT project (ANR-17-CE05-0005-01).

\section{Corresponding authors}

SB: shuming.bai@univ-amu.fr

MB: mario.barbatti@univ-amu.fr, www.barbatti.org 


\section{References}

1. S. W. Pridgeon, R. Heer, G. A. Taylor, D. R. Newell, K. O'Toole, M. Robinson, Y. Z. Xu, P. Karran and A. V. Boddy, Br. J. Cancer, 2011, 104, 1869-1876.

2. A. Massey, Y.-Z. Xu and P. Karran, Curr. Biol., 2001, 11, 1142-1146.

3. X. Zhang, G. Jeffs, X. Ren, P. O’Donovan, B. Montaner, C. M. Perrett, P. Karran and Y.-Z. Xu, DNA Repair, 2007, 6, 344-354.

4. S. Euvrard, J. Kanitakis and A. Claudy N. Engl. J. Med., 2003, 348, 16811691.

5. K. Komeda, S. Iwamoto, S. Kominami and T. Ohnishi, Photochem. Photobiol, 1997, 65, 115-118.

6. M. Barbatti, A. J. A. Aquino, J. J. Szymczak, D. Nachtigallova, P. Hobza and H. Lischka, Proc. Natl. Acad. Sci. USA, 2010, 107, 21453-21458.

7. L. Serrano-Andres and M. Merchan, J. Photochem. Photobiol. C-Photochem. Rev., 2009, 10, 21-32.

8. S. Mai, M. Pollum, L. Martínez-Fernández, N. Dunn, P. Marquetand, I. Corral, C. E. Crespo-Hernández and L. González, Nat. Commun., 2016, 7, 13077.

9. M. Pollum, S. Jockusch and C. E. Crespo-Hernández, J. Am. Chem. Soc., 2014, 136, 17930-17933.

10. S. M. Bai and M. Barbatti, J. Phys. Chem. A, 2016, 120, 6342-6350.

11. C. Reichardt and C. E. Crespo-Hernandez, J. Phys. Chem. Lett., 2010, 1, 22392243.

12. B. A. West, J. M. Womick and A. M. Moran, J. Phys. Chem. A, 2013, 117, $5865-5874$. 
13. K. Taras-Goślińska, G. Burdziński and G. Wenska, J. Photochem. Photobiol. A, 2014, 275, 89-95.

14. B. Ashwood, S. Jockusch and C. E. Crespo-Hernandez, Molecules, 2017, 22, 15.

15. C. Reichardt, C. Guo and C. E. Crespo-Hernandez, J. Phys. Chem. B, 2011, 115, 3263-3270.

16. J. Jiang, T.-s. Zhang, J.-d. Xue, X. Zheng, G. Cui and W.-h. Fang, J. Chem. Phys., 2015, 143, 175103.

17. S. Mai, P. Marquetand and L. González, J. Phys. Chem. A, 2015, 119, 95249533.

18. G. Cui and W. Thiel, J. Phys. Chem. Lett., 2014, 5, 2682-2687.

19. J. P. Gobbo and A. C. Borin, Comput. Theor. Chem., 2014, 1040-1041, 195201.

20. G. Cui and W.-h. Fang, J. Chem. Phys., 2013, 138, 044315.

21. S. Mai, P. Marquetand and L. Gonzalez, J. Phys. Chem. Lett., 2016, 7, 19781983.

22. L. Martinez-Fernandez, I. Corral, G. Granucci and M. Persico, Chem. Sci., $2014, \mathbf{5}, 1336-1347$.

23. C. Schweitzer, Z. Mehrdad, A. Noll, E. W. Grabner and R. Schmidt, J. Phys. Chem. A, 2003, 107, 2192-2198.

24. H. Kuramochi, T. Kobayashi, T. Suzuki and T. Ichimura, J. Phys. Chem. B, 2010, 114, 8782-8789.

25. S. Bai and M. Barbatti, Phys. Chem. Chem. Phys., 2017, 19, 12674-12682.

26. S. Bai and M. Barbatti, J. Chem. Theory Comput., 2017, 13, 5528-5538. 
27. S. Bai and M. Barbatti, J. Phys. Chem. Lett., 2017, 8, 5456-5460.

28. J. Finley, P. A. Malmqvist, B. O. Roos and L. Serrano-Andrés, Chem. Phys. Lett., 1998, 288, 299-306.

29. W. J. Hehre, R. Ditchfield. and J. A. Pople, J. Chem. Phys., 1972, 56, $2257-$ 2261.

30. A. D. Becke, J. Chem. Phys., 1993, 98, 5648-5652.

31. X. Xu and W. A. Goddard, Proc. Natl. Acad. Sci. USA, 2004, 101, 2673-2677.

32. J.-D. Chai and M. Head-Gordon, Phys. Chem. Chem. Phys., 2008, 10, 66156620.

33. M. J. Frisch, G. W. Trucks, H. B. Schlegel, G. E. Scuseria, M. A. Robb, J. R. Cheeseman, G. Scalmani, V. Barone, B. Mennucci, G. A. Petersson, H. Nakatsuji, M. Caricato, X. Li, H. P. Hratchian, A. F. Izmaylov, J. Bloino, G. Zheng, J. L. Sonnenberg, M. Hada, M. Ehara, K. Toyota, R. Fukuda, J. Hasegawa, M. Ishida, T. Nakajima, Y. Honda, O. Kitao, H. Nakai, T. Vreven, J. A. Montgomery Jr., J. E. Peralta, F. Ogliaro, M. Bearpark, J. J. Heyd, E. Brothers, K. N. Kudin, V. N. Staroverov, R. Kobayashi, J. Normand, K. Raghavachari, A. Rendell, J. C. Burant, S. S. Iyengar, J. Tomasi, M. Cossi, N. Rega, J. M. Millam, M. Klene, J. E. Knox, J. B. Cross, V. Bakken, C. Adamo, J. Jaramillo, R. Gomperts, R. E. Stratmann, O. Yazyev, A. J. Austin, R. Cammi, C. Pomelli, J. W. Ochterski, R. L. Martin, K. Morokuma, V. G. Zakrzewski, G. A. Voth, P. Salvador, J. J. Dannenberg, S. Dapprich, A. D. Daniels, Ö. Farkas, J. B. Foresman, J. V. Ortiz, J. Cioslowski and D. J. Fox, Gaussian 09, Revision A.1, Gaussian Inc., Wallingford CT, 2009.

34. S. G. Chiodo and N. Russo, J. Comput. Chem., 2009, 30, 832-839. 
35. X. Gao, S. M. Bai, D. Fazzi, T. Niehaus, M. Barbatti and W. Thiel, J. Chem. Theory Comput., 2017, 13, 515-524.

36. M. Casida, in Recent advances in density functional methods, Part I, ed. D. Chong, World Scientific, Singapore, 1995, pp. 155-192.

37. L. Rao, H. W. Ke, G. Fu, X. Xu and Y. J. Yan, J. Chem. Theory Comput., 2009, 5, 86-96.

38. O. B. Roos, V. Veryazov and P.-O. Widmark, Theor. Chem. Acc., 2003, 111, 345-351.

39. G. Ghigo, B. O. Roos and P.-A. Malmqvist, Chem. Phys. Lett., 2004, 396, $142-$ 149.

40. B. O. Roos and K. Andersson, Chem. Phys. Lett., 1995, 245, 215-223.

41. B. G. Levine, J. D. Coe and T. J. Martínez, J. Phys. Chem. B, 2008, 112, 405413

42. Q. Ou and J. E. Subotnik, J. Phys. Chem. C, 2013, 117, 19839-19849.

43. M. A. Palafox and N. Iza, J. Mol. Struct., 2012, 1028, 181-195.

44. Y. P. Yurenko, R. O. Zhurakivsky, M. Ghomi, S. P. Samifflenko and D. M. Hovorun, J. Phys. Chem. B, 2008, 112, 1240-1250.

45. P. Zhou, F. F. Tian, F. L. Lv and Z. C. Shang, Proteins-Structure Function and Bioinformatics, 2009, 76, 151-163.

46. H. S. Biswal, P. R. Shirhatti and S. Wategaonkar, J. Phys. Chem. A, 2009, 113, 5633-5643.

47. H. Kuramochi, T. Kobayashi, T. Suzuki and T. Ichimura, J. Phys. Chem. B, 2010, 114, 8782-8789. 
48. J. J. Zheng, T. Yu, E. Papajak, I. M. Alecu, S. L. Mielke and D. G. Truhlar, Phys. Chem. Chem. Phys., 2011, 13, 10885-10907.

49. L. Martinez-Fernandez, L. Gonzalez and I. Corral, Chem. Commun., 2012, 48, 2134-2136. 
TOC graphic

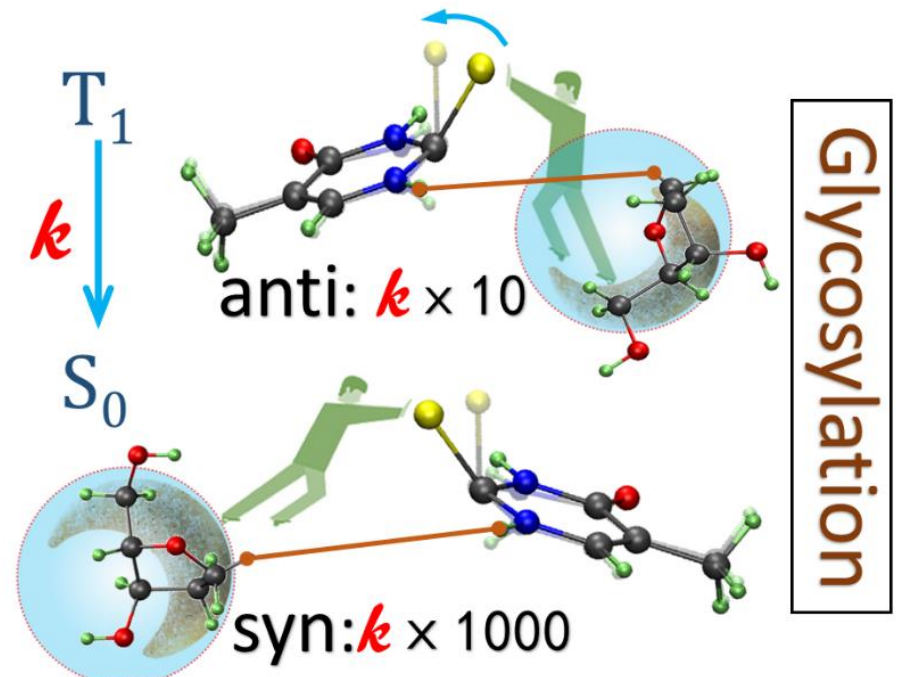

Functionalization of the sugar group can be used to control the triplet decay rate of thionucleosides. 